\title{
Quality of life, characteristics and survival of patients with HIV and lymphoma
}

\author{
Catherine Diamond - Thomas H. Taylor • \\ Hoda Anton-Culver
}

Accepted: 1 December 2009/Published online: 8 January 2010

(C) The Author(s) 2010. This article is published with open access at Springerlink.com

\begin{abstract}
Purpose We sought to compare the quality of life (QOL), characteristics, and survival of patients with non-Hodgkin lymphoma (NHL) with and without human immunodeficiency virus (HIV) infection.

Methods Using the population-based cancer registry for Orange and San Diego Counties, We recruited 50 patients with HIV and systemic NHL (cases) and 50 age, sex and race-matched NHL patients without HIV (controls) diagnosed with NHL during 2002-2006. Patients completed a medical history survey and QOL instrument, the Functional Assessment of Human Immunodeficiency Virus Infection (FAHI) for cases and Functional Assessment of Cancer Therapy-General (FACT G) for controls.

Results HIV-infected patients had worse overall QOL and survival than uninfected patients. QOL differences were more marked in the areas of functional, physical and social well-being than in the area of emotional well-being. HIV-infected patients had lower income and were less likely to have private insurance and more likely to have diffuse large B cell histology than uninfected patients.

Conclusion HIV-infected NHL patients had worse QOL and survival than uninfected patients, due to a combination of co-morbidity, aggressive histology and lack of social support. However, their emotional well-being was comparable to that of uninfected NHL patients and better than historical norms for the HIV-infected.
\end{abstract}

C. Diamond $(\bowtie) \cdot$ T. H. Taylor · H. Anton-Culver Department of Epidemiology, University of California Irvine, Irvine, CA 92697-7555, USA

e-mail: diamondc@uci.edu

C. Diamond

Department of Medicine, University of California Irvine, Irvine, CA 92697-7555, USA
Keywords HIV · AIDS - Quality of life · Non-Hodgkin lymphoma $\cdot$ FAHI $\cdot$ FACT G

\begin{tabular}{|c|c|}
\hline \multicolumn{2}{|c|}{ Abbreviations } \\
\hline AIDS & Acquired immunodeficiency syndrome \\
\hline DLBCL & Diffuse large B cell lymphoma \\
\hline FACT G & $\begin{array}{l}\text { Functional assessment of cancer therapy- } \\
\text { general }\end{array}$ \\
\hline FAHI & $\begin{array}{l}\text { Functional assessment of human } \\
\text { immunodeficiency virus infection }\end{array}$ \\
\hline GLM & General linear model \\
\hline G-CSF & Granulocyte colony-stimulating factor \\
\hline HAART & Highly active antiretroviral therapy \\
\hline HIV & Human immunodeficiency virus \\
\hline ICD-O & $\begin{array}{l}\text { International classification of diseases for } \\
\text { oncology }\end{array}$ \\
\hline MANOVA & Multivariate analysis of variance \\
\hline NHL & Non-Hodgkin lymphoma \\
\hline QOL & Quality of life \\
\hline SD & Standard deviation \\
\hline
\end{tabular}

\section{Introduction}

Non-Hodgkin lymphoma (NHL) has been a major source of morbidity and mortality among individuals with human immunodeficiency virus (HIV) since the first description of acquired immunodeficiency syndrome (AIDS) in the early 1980s [1]. Regarding lymphoma patients without HIV, an increased focus on quality of life (QOL) has emerged [2]. However, there are few studies of HIV-associated NHL that focus on QOL. We are aware of a single paper reporting QOL among HIV-infected patients who received 
oral combination chemotherapy with filgrastim (granulocyte colony-stimulating factor (G-CSF) [3]. Indeed, a recent Cochrane review of treatment of AIDS-related NHL specifically requested more trials with QOL as an endpoint [4]. Addressing this knowledge gap, we performed a casecontrol study comparing QOL as well as clinical characteristics between patients with HIV and NHL and NHL without HIV infection.

Since the introduction of highly active antiretroviral therapy (HAART) in the late 1990s, survival among patients with AIDS-related NHL has improved dramatically [5]. While physicians 10 years ago were hesitant to treat patients with AIDS and NHL with chemotherapy, contemporary patients typically receive the same aggressive chemotherapy regimens that patients without AIDS receive [6]. Despite these therapeutic improvements, we hypothesized that contemporary patients with HIV infection and NHL would have worse QOL than contemporary uninfected patients with NHL and historical HIV-infected patients.

HIV-infected NHL patients differ from NHL patients without HIV infection. They are typically younger and more likely to be male and racial/ethnic minorities [7]. Because of these demographic differences, we matched by age, race and sex in comparing NHL patients with and without HIV-infection. Also, HIV-infected individuals (with or without concurrent NHL) are more likely to be poor, publicly insured, and illicit drug users than uninfected individuals [8]. Because of the social stigma associated with HIV and its associated sexual and drug-related risk factors, the HIV-infected may feel rejected by their families and society as a whole. Compared with uninfected NHL patients, those with concurrent HIV and NHL have an additional co-morbidity which requires medication and may have caused pre-existing disability. NHL histology among the HIV-infected typically is more aggressive than among patients without HIV [1]. Because of these social and biologic differences between NHL patients with and without HIV infection, we anticipated that we would find a lower QOL among the HIV-infected NHL patients in all categories of well-being measured: emotional, social, physical and functional.

\section{Methods}

The University of California Irvine Institutional Review Board approved the study prior to initiation (\#2002-2428) in accordance with the 1964 Declaration of Helsinki. We recruited 100 residents of Orange or San Diego Counties diagnosed with systemic NHL from 2002-2006, 50 HIVinfected and 50 without HIV diagnoses. We identified subjects through the California cancer registry for Orange and San Diego Counties and provider referrals. We sought to contact patients within 1 year of their NHL diagnosis. Prior to contacting a patient, we sent a letter to the reporting physician to determine if there was any reason to not contact the patient. If there were no contraindications, we sent a packet with the consent form, medical record release form, medical history survey and QOL instrument to the potential subject along with a cover letter explaining the study. If the potential subject did not return the signed consent and surveys, a research associate telephoned the subject to answer questions, encourage participation and offer assistance completing the packet.

During 2002-2006, the California Cancer Registry for Orange and San Diego Counties contained 171 patients flagged as HIV-infected (potential cases) and 4,932 patients with systemic NHL flagged as HIV-seronegative or sero-unknown (potential controls). In previous research we have demonstrated this HIV flag to be $97 \%$ sensitive and $92 \%$ specific [9]. Of the 171 potential cases, we recruited 51 . Thirty potential cases died prior to contact and we were unable to make contact with 15 ; one was too ill to participate. The remaining 74 potential cases were reported to the Cancer Registry too late to be eligible for the study (more than 1 year after NHL diagnosis). We excluded one case from analysis because we did not have a control for him. We attempted to contact 130 controls with an initial goal of a 2:1 case:control ratio. Seven potential controls died prior to contact; we were unable to make contact with 37 and 16 declined participation. Although we recruited 70 controls, we selected the 50 sex and racematched controls with complete data that matched the cases by age ( \pm 5 years) in order to achieve a $1: 1$ case:control ratio. Thus, the analysis included 50 cases and 50 controls.

The QOL instrument was the Functional Assessment of Human Immunodeficiency Virus Infection (FAHI) in patients with HIV and the Functional Assessment of Cancer Therapy-General (FACT G) in patients without HIV $[10,11]$. These QOL instruments have been validated in English and Spanish [12,13] and all patient forms and surveys were available in both languages. The FAHI contains the entire FACT $\mathrm{G}$ and additional items reflecting HIV/AIDS specific concerns. This allows cross-disease comparison of QOL through calculation of an HIV specific score and a FACT G score derived from the FAHI. A SAS (Cary, NC) program is available that calculates these scores [13].

We used data from California Cancer Registry as of April, 2008 to obtain information on chemotherapy, histology, site and stage of NHL. We used the classification system of the International Classification of Diseases for Oncology (ICD-O Third Edition) [14] to divide the histology into three categories: diffuse large B cell lymphoma 
(DLBCL, code 9680 and 9684) follicular (codes 9690, 9691, 9695, and 9698) and other. We abstracted CD4 cell counts and viral load results drawn within 3 months of NHL diagnosis (but prior to chemotherapy) from the medical record.

\section{Statistical methods}

We used SAS 9.2 for all statistical analyses. We performed the chi-square test to compare proportions between cases and controls. We used the $t$-test to compare mean QOL scores between cases with or without a diagnosis of HIV prior to NHL diagnosis and time between NHL diagnosis and study entry between cases and controls. We used the General Linear Model (GLM) procedure to compare unadjusted quality of life scores between cases and controls. Although we matched cases and controls on age, race and sex, cases and controls differed socioeconomically and biologically. To account for correlations among the QOL subscales, we performed multivariate analysis of variance (MANOVA) adjusting for all socioeconomic and biologic variables that differed significantly between cases and controls (Table 1). We employed the Kaplan-Meier method to calculate survival. All tests are two-sided with $p<.05$ considered statistically significant.

\section{Results}

Demographics

The sex distribution was $96 \%$ male (48 men and two women in each matched group). There were 26 whites (52\%), 21 Latinos (42\%), two African Americans (4\%) and one Asian (2\%) in each group. The median age at NHL diagnosis was 45 years (range 17-70). Controls were more likely than cases to have private insurance $(p<.03$, Table 1) and cases were more likely than controls to have an annual income less than $\$ 20,000(p<.001)$.

\section{Clinical characteristics}

HIV-infected individuals were more likely to have diffuse large B cell lymphoma than controls and controls were more likely to have follicular lymphoma than cases $(p<.001$, Table 1$)$. HIV-infected patients also were more likely than uninfected patients to have extranodal lymphoma $(p<.02)$. There was no statistical difference in NHL stage $(p<.59)$ or use of chemotherapy $(p<.78)$ between the two groups. Among the 50 cases, $24(48 \%)$ had prior AIDS (diagnosed either by opportunistic infection or CD4 $<200 / \mathrm{mcl})$ and $15(30 \%)$ did not have AIDS
Table 1 Characteristics of Non-Hodgkin Lymphoma (NHL) patients with and without Human Immunodeficiency Virus (HIV) infection, residing in Orange and San Diego Counties, 2002-2006

\begin{tabular}{|c|c|c|c|}
\hline & $\begin{array}{l}\text { HIV-NHL }(N=50) \\
N(\text { column } \%)\end{array}$ & $\begin{array}{l}\text { NHL }(N=50) \\
N(\text { column } \%)\end{array}$ & $p$ by $\chi^{2}$ \\
\hline Insurance & & & .03 \\
\hline $\begin{array}{l}\text { Government including Medicaid, Medicare, } \\
\text { county, veteran, military, and Ryan White }\end{array}$ & $27(54)$ & $14(28)$ & \\
\hline Private & $14(28)$ & $21(42)$ & \\
\hline Self pay/declined to state/do not know & $9(18)$ & $15(30)$ & \\
\hline Income & & & .001 \\
\hline Less than $\$ 20,000$ annual & $23(46)$ & $9(18)$ & \\
\hline$\$ 20,000$ or more annual & $18(36)$ & $37(74)$ & \\
\hline Declined to state & $9(18)$ & $4(8)$ & \\
\hline Histology & & & .001 \\
\hline Diffuse large B cell (including immunoblastic) & $35(70)$ & $19(38)$ & \\
\hline Follicular & $0(0)$ & $17(34)$ & \\
\hline Other & $15(30)$ & $14(28)$ & \\
\hline Nodal status & & & .02 \\
\hline Nodal & $34(68)$ & $44(88)$ & \\
\hline Extranodal & $16(32)$ & $6(12)$ & \\
\hline Stage $e^{\mathrm{a}}$ & & & .59 \\
\hline Stage I-III & $18(37)$ & $21(42)$ & \\
\hline Stage IV & $31(63)$ & $29(58)$ & \\
\hline Chemotherapy & & & .78 \\
\hline Yes & $43(86)$ & $42(84)$ & \\
\hline No & 7 (14) & $8(16)$ & \\
\hline
\end{tabular}


before NHL diagnosis; the AIDS history of the remaining $11(22 \%)$ was unknown. Among 38 HIV-infected patients with recorded laboratory values within 3 months of NHL diagnosis yet prior to chemotherapy, the median CD4 cell count was $157 / \mathrm{mcl}$ (range 15-750) and median HIV RNA was 18,600 copies/ml (log 4.3) (range $<50-880,000$ ). Although over half (54\%) had a CD4 cell count $<200 / \mathrm{mcl}$, more than one-third (34\%) had an HIV RNA of $<400$ copies $/ \mathrm{ml}$.

\section{Medical history survey}

All subjects completed medical history and QOL surveys a median 8 months after NHL diagnosis (range 0-16). Cases completed the survey a median of 4 months after NHL diagnosis (range 0-15) and controls completed the survey a median of 11 months after NHL diagnosis (range 3-16) $(p<.001)$. Among 42 patients who recalled when they were first diagnosed with HIV infection, the median duration of infection was 9 years (ranging from diagnosed 1 month after NHL diagnosis to 24 years prior to NHL diagnosis). Among all 50 cases, $11(22 \%)$ said they were newly diagnosed with HIV. Twenty-eight $(56 \%)$ reported that they were taking HAART at the time of NHL diagnosis; $17(34 \%)$ stated they were not taking HAART and five $(10 \%)$ did not know. By the time of survey completion,

Table 2 Quality of life in patients with Non-Hodgkin Lymphoma (NHL) with and without Human Immunodeficiency Virus Infection (HIV) as measured by the Functional Assessment of Human
$39(78 \%)$ were on HAART, seven (14\%) were not and four $(8 \%)$ did not know.

Quality of life differences between NHL patients with and without HIV Infection

NHL patients with HIV infection had worse overall QOL than NHL patients without HIV infection $(p<.0001$, Table 2). These differences were more marked $(p<.005$ by GLM) in the areas of functional, physical and social well-being than in the area of emotional well-being. In the MANOVA model adjusted for demographic and clinical factors which were statistically significantly different between cases and controls (nodal status and histologic, insurance and income categories shown in Table 1), emotional well-being did not statistically differ between NHL patients with and without HIV infection. NHL patients without HIV infection had similar QOL scores to previously reported values for cancer patients [15].

Quality of life among HIV-infected NHL patients versus reported HIV-infected patient norm

Table 3 shows QOL scores for HIV-infected patients as measured by the FAHI. The overall QOL of current patients with HIV-associated NHL is similar to that of

Immunodeficiency Virus Infection (FAHI) and Functional Assessment of Cancer Therapy-General (FACT-G)

\begin{tabular}{|c|c|c|c|c|c|}
\hline & $\begin{array}{l}\text { HIV-NHL }(N=50) \\
\text { mean }(\mathrm{SD})\end{array}$ & $\begin{array}{l}\text { NHL }(N=50) \\
\text { mean }(\mathrm{SD})\end{array}$ & $\begin{array}{l}p \text { by } \mathrm{GLM}^{\mathrm{a}} \text { comparing mean } \\
\text { in HIV-infected NHL versus } \\
\text { uninfected NHL }\end{array}$ & $\begin{array}{l}p \text { by MANOVA }{ }^{\mathrm{b}} \text { comparing } \\
\text { mean in HIV-infected NHL } \\
\text { versus uninfected NHL }\end{array}$ & $\begin{array}{l}\text { Reported cancer } \\
\text { patient norm }{ }^{c} \\
(N=2,236)\end{array}$ \\
\hline Total & $68.4(15.9)$ & $81.9(16.1)$ & .0001 & .0001 & $80.9(17.0)$ \\
\hline Emotional well-being & $17.3(4.1)$ & $18.9(3.8)$ & .05 & 0.19 & $18.7(4.5)$ \\
\hline Functional well-being & $14.6(6.2)$ & $18.8(7.0)$ & .002 & .0001 & $18.9(6.8)$ \\
\hline Physical well-being & $18.0(6.7)$ & $22.3(5.9)$ & .001 & .002 & $21.3(6.0)$ \\
\hline Social well-being & $18.5(6.5)$ & $21.9(5.1)$ & .005 & .03 & $22.1(5.3)$ \\
\hline
\end{tabular}

a General linear model

b Multivariate analysis of variance model includes nodal status and histologic, insurance and income categories

${ }^{\mathrm{c}}$ Normative data presented for comparison [15]

Table 3 Quality of life in patients with Non-Hodgkin Lymphoma (NHL) and Human Immunodeficiency Virus Infection (HIV) as measured by the Functional Assessment of HIV Infection quality of life instrument (FAHI)

\begin{tabular}{llc}
\hline & $\begin{array}{l}\text { HIV and NHL } \\
(N=50) \text { mean }(S D)\end{array}$ & $\begin{array}{l}\text { Reported HIV-infected patient } \\
\text { norm [13] }(N=352) \text { mean }(S D)\end{array}$ \\
\hline Total mean (SD) & $110.0(26.5)$ & $106.3(26.0)$ \\
Emotional well-being mean (SD) & $25.7(9.3)$ & $20.4(8.6)$ \\
Functional well-being mean (SD) & $29.6(9.9)$ & $32.2(9.7)$ \\
Physical well-being mean (SD) & $24.6(10.0)$ & $24.2(9.5)$ \\
Social well-being mean (SD) & $21.6(7.3)$ & $21.4(6.7)$ \\
Cognitive functioning mean (SD) & $8.5(2.5)$ & $8.1(2.9)$ \\
\hline
\end{tabular}


Fig. 1 Survival Comparison between 50 patients with Human Immunodeficiency Virus (HIV) Infection and NonHodgkin Lymphoma (NHL) and 50 patients with NHL without HIV, 2002-2007, Orange and San Diego Counties

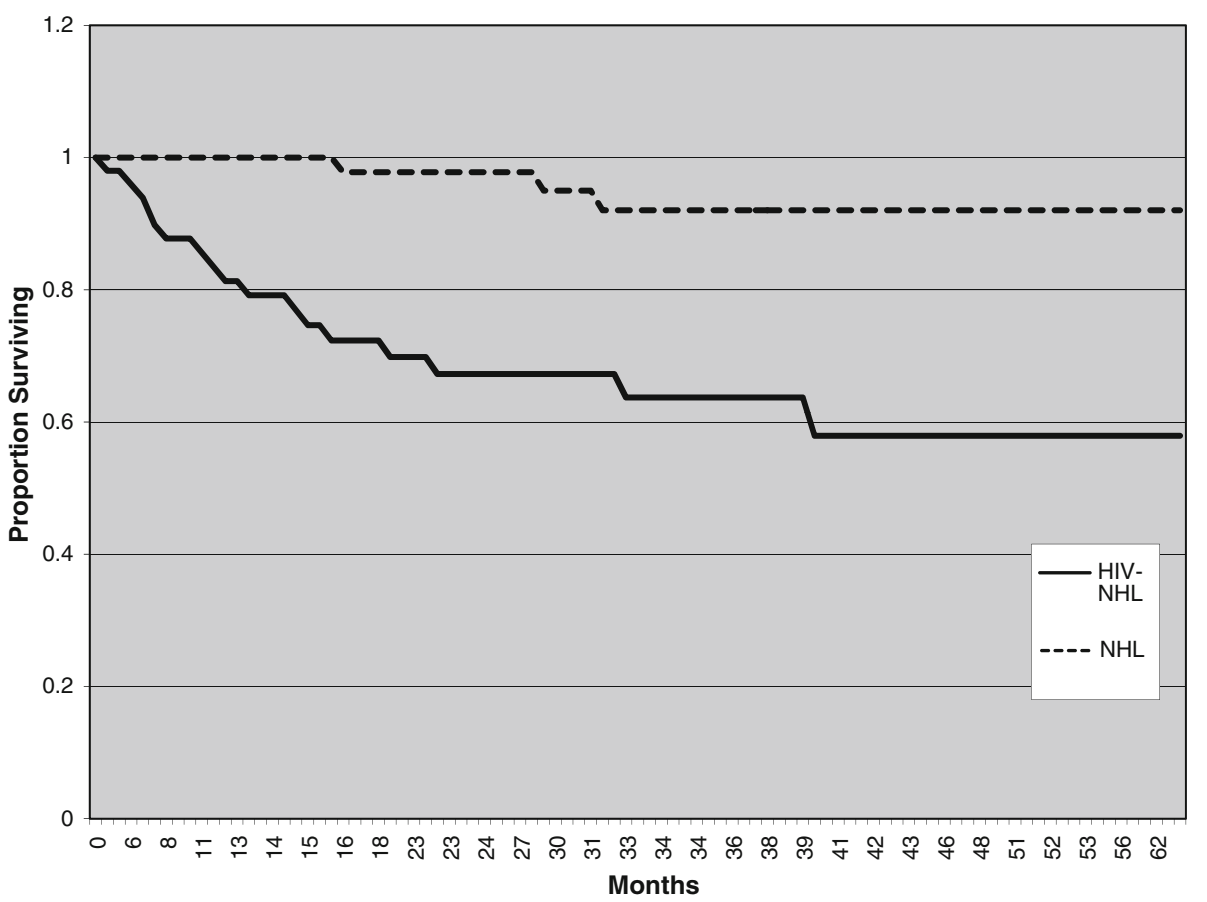

historical HIV-infected patients without NHL [13]. The emotional well-being score was higher for contemporary HIV-infected patients with NHL than reported norms for HIV-infected patients. The functional well-being score was slightly lower for HIV-infected patients with NHL than historical patients. Physical and social well-being and cognitive functioning scores were similar to reported norms. QOL scores (total and subscale) did not differ between cases with prior HIV diagnoses and those who were newly diagnosed $(p>.05)$.

\section{Survival}

The median follow up time was 33 months (range 266 months). Among the 50 cases, 17 died during followup. Among the 50 controls, two died during follow-up. Thus, survival among cases was worse than among controls $(p<.001)$. Because more than half of the subjects were alive at most recent follow-up in both groups, it is impossible to calculate a median survival (Fig. 1).

\section{Discussion}

Despite the availability of antiretrovirals and chemotherapy, HIV-infected patients had worse overall QOL and survival than uninfected patients with NHL. We anticipated that we would find a lower quality of life among the HIVinfected NHL patients in all categories of well-being, physical and functional as well as social and emotional.
However, we found that worse QOL among the HIVinfected was due to lower functional, physical and social well-being rather than emotional well-being (Table 2). HIV-infected patients were more likely to have diffuse large B cell and extranodal lymphoma (Table 1). These biologic differences between NHL patients with and without HIV infection combined with the debility and medication management associated with concurrent HIV infection may explain the lower physical QOL subscale scores among the HIV-infected. HIV-infected patients had less income and were less likely to have private insurance than uninfected patients (Table 1), possibly contributing to a lower functional QOL even prior to NHL diagnosis.

The low social well-being scores for the HIV-infected may reflect lack of familial or partner support. HIVinfected individuals may be estranged from their families because of relatives' concerns regarding risk factors for HIV (e.g. homosexuality or injection drug use) or fear and prejudice. Lack of partner support may be due to the death or illness of an HIV-infected partner or lack of societal support for same sex unions.

There was no statistical difference in emotional wellbeing between cases and controls in the adjusted model. The issues reflected in the emotional well-being score (such as anxiety, hopelessness and fear of death) may be equally concerning to both cases and controls. Alternatively, some HIV-infected patients may have addressed these issues in the past at the time of HIV diagnosis and have become accustomed to living with them. We discuss below how contemporary patients with HIV and NHL have 
better emotional well-being subscale scores than historical norms for HIV patients, suggesting that the availability of HAART has influenced this subscale score in particular.

Although we initially hypothesized that contemporary patients with HIV-infection and NHL would have worse QOL than historical HIV-infected patients, we were surprised to find that the overall QOL of our patients with HIV-associated NHL was similar to that of historical HIVinfected patients without NHL as measured by the FAHI (Table 3) [13]. These QOL norms for HIV-infected patients are from the pre-HAART era and reflect the poor prognosis and stigma associated with AIDS of that time. Contemporary patients with HIV-associated NHL have better emotional well-being than historical HIV-infected patients, perhaps due to increased optimism about prognosis due to the availability of HAART. The survival of patients with HIV-associated NHL was better in our study than historical figures [1], also reflecting the benefit of HAART

While there was no difference between contemporary and historical patients in the physical, social and cognitive subscales, the functional well-being subscale score was more than two points worse in contemporary HIV-infected patients with NHL than among historical HIV-infected patients but the difference was less than 0.5 standard deviation (SD). In the interpretation of differences in health-related quality of life, an effect size of $0.5 \mathrm{SD}$ is a relatively stable important difference [16]. The developers of the FACT-G and FAHI consider a five point difference in overall score and a two point difference in subscale score as clinically meaningful for cancer patients [15]. A decrease in functional well being could be due to the effect of concurrent NHL and its treatment but might be mitigated by antiretroviral therapy.

Beyond the benefit of HAART, designing an intervention to improve the QOL of patients with HIV and NHL is not simple. Chemotherapy regimens of shorter duration with fewer side effects and oral administration would improve QOL. In-home support, both physical and social, to bridge the period of decreased function would improve QOL. While home health care for hygiene requires inperson assistance, perhaps social support could be provided by telephone. Given that AIDS-related NHL is relatively uncommon (390 cases per 100,000 person-years among people with AIDS in 1996-2002) [17], there would be few people able to participate in support groups for this illness. Interventions in physician offices, infusion centers or hospitals during routine care would be convenient for patients. Since most physicians and nurses lack the training and time to intervene, social workers or psychologists might play a role. Given that many patients with HIV-related NHL are poor and publicly insured, it is unclear how to cover the costs of any intervention.
This manuscript is unique in that it is the only study, to our knowledge, to use information from the cancer registry to identify and contact patients with a HIV-related malignancy. There was a population-based case-control study of NHL using rapid case ascertainment in the San Francisco Bay Area between 1988 and 1995. In that study, all subjects received a questionnaire inquiring about their HIV status and men had the option to participate in a laboratory portion of the study that included HIV serologic testing [18]. The standardized and validated QOL instruments employed in the study enhance the value of our findings $[10-13,15]$.

Our study is limited by the moderate number of subjects. The high morbidity and mortality associated with AIDS and lymphoma as well as the social and economic issues discussed above made it challenging to contact and followup cases. We also had difficulty finding controls matched by age and race/ethnicity. Because there is no routine system for rapid case ascertainment and we were afraid of patients dying prior to study participation, we did not standardize the time to interview. Since medical records were scattered between HIV specialist and oncologist offices and various hospitals, our information regarding prior AIDS diagnoses and current CD4 cell counts is incomplete. Some patients declined to answer survey questions regarding their income and insurance, also resulting in incomplete data.

Since some potential subjects died prior to contact, there is bias toward improved survival in our HIV-infected study participants compared with all HIV-infected patients with lymphoma. The HIV-infected patients who participated were healthy enough to give consent and complete the surveys and thus were not representative of all HIVinfected patients with NHL. Another source of bias is the different NHL histology between cases and controls. However, given the inherent imbalance in histologic pattern between the HIV-infected and uninfected, it was not feasible to match on histology as well as age, sex, and race/ ethnicity. Although we used the population-based cancer registry to recruit, we matched our controls to cases; the controls are not representative of all patients with systemic NHL who would typically be older and include more women than our controls. HIV infection in the United States is more common in men than women, so our study included few women [7].

Quality of the life among patients with lymphoma is an important yet understudied topic. We conclude that even among the HIV-infected lymphoma patients most likely to do well, QOL and survival lag behind that of uninfected patients. However, emotional QOL has improved compared to historical HIV-infected patient norms. Survival among HIV-infected patients with lymphoma also is better than in the past. Despite the widespread availability of 
HIV-testing, undiagnosed individuals present with advanced disease [19]. Similarly, not all HIV-infected patients access antiretroviral therapy. The low CD4 cell counts and elevated viral loads of our patients suggest uncontrolled HIV infection and issues regarding antiretroviral access, adherence, tolerance and/or resistance. Therefore, NHL will continue to be a source of morbidity and mortality among the HIV-infected. Our study provides a foundation for future research.

Acknowledgments We greatly appreciate the efforts of the patients who participated and physicians who supported our research, especially Dr. Stephen Baird. We are grateful for technical support from Archana Kulkarni, Maureen Gildea-Schroeder, Ed Barber and Georgiana Margarit. We also thank our exceptional research assistants Jeanette Overton and Katherine Miller. The work was funded by National Institutes of Health K07 CA96480 and University AIDS Research Program/California HIV Research Program California Collaborative Treatment Group grants CC02-SD-003, CH05-SD-607, CH07-SD-607.

Open Access This article is distributed under the terms of the Creative Commons Attribution Noncommercial License which permits any noncommercial use, distribution, and reproduction in any medium, provided the original author(s) and source are credited.

\section{References}

1. Coté, T. R., Biggar, R. J., Rosenberg, P. S., Devesa, S. S., Percy, C., Yellin, F. J., et al. (1997). Non-Hodgkin's lymphoma among people with AIDS: Incidence, presentation and public health burden. AIDS/cancer study group. International Journal of Cancer, 73, 645-650.

2. Bellizzi, K. M., Rowland, J. H., Arora, N. K., Hamilton, A. S., Miller, M. F., \& Aziz, N. M. (2009). Physical activity and quality of life in adult survivors of non-Hodgkin's lymphoma. Journal of Clinical Oncology, 27, 960-966.

3. Remick, S. C., Sedransk, N., Haase, R. F., Blanchard, C. G., Ramnes, C. R., Nazeer, T., et al. (2001). Oral combination chemotherapy in conjunction with filgrastim (G-CSF) in the treatment of AIDS-related non-Hodgkin's lymphoma: Evaluation of the role of G-CSF; quality-of-life analysis and long-term followup. American Journal of Hematology, 66, 178-188.

4. Martí-Carvajal, A. J., Cardona, A. F., Lawrence, A. (2009). Interventions for previously untreated patients with AIDS-associated Non-Hodgkin's Lymphoma. Cochrane Database of Systematic Reviews, 3, CD005419.

5. Diamond, C., Taylor, T. H., Aboumrad, T., \& Anton-Culver, H. (2006). Changes in acquired immunodeficiency syndrome-related non-Hodgkin lymphoma in the era of highly active antiretroviral therapy: Incidence, presentation, treatment, and survival. Cancer, 106, 128-135.
6. Boué, F., Gabarre, J., Gisselbrecht, C., Reynes, J., Cheret, A., Bonnet, F., et al. (2006). Phase II trial of CHOP plus rituximab in patients with HIV-associated non-Hodgkin's lymphoma. Journal of Clinical Oncology, 24, 4123-4128.

7. Centers for Disease Control and Prevention (2009). HIV/AIDS surveillance report, 2007. Atlanta: US Department of Health and Human Services, Centers for Disease Control and Prevention, 19: 12.

8. Goldman, D. P., Leibowitz, A. A., Joyce, G. F., Fleishman, J. A., Bozzette, S. A., Duan, N., et al. (2003). Insurance status of HIVinfected adults in the post-HAART era: evidence from the United States. Applied Health Economics and Health Policy, 2, 85-90.

9. Diamond, C., Taylor, T. H., Im, T., Wallace, M., Saven, A., \& Anton-Culver, H. (2007). How valid is using cancer registries' data to identify acquired immunodeficiency syndrome-related nonHodgkin's lymphoma? Cancer Causes and Control, 18, 135-142.

10. Cella, D. F., McCain, N. L., Peterman, A. H., Mo, F., \& Wolen, D. (1996). Development and validation of the Functional assessment of human immunodeficiency virus infection (FAHI) quality of life instrument. Quality of Life Research, 5, 450-463.

11. Cella, D. F., Tulsky, D. S., Gray, G., Sarafian, B., Linn, E., Bonomi, A., et al. (1993). The functional assessment of cancer therapy scale: Development and validation of the general measure. Journal of Clinical Oncology, 11, 570-579.

12. Cella, D., Hernandez, L., Bonomi, A. E., Corona, M., Vaquero, M., Shiomoto, G., et al. (1998). Spanish language translation and initial validation of the functional assessment of cancer therapy quality-of-life instrument. Medical Care, 36, 1407-1418.

13. Peterman, A. H., Cella, D., Mo, F., \& McCain, N. (1997). Psychometric validation of the revised Functional assessment of human immunodeficiency virus infection (FAHI) quality of life instrument. Quality of Life Research, 6, 572-584.

14. Fritz, A., Percy, C., Jack, A., Kanagaratnam, S., Sobin, L., Parkin, D. M., et al. (2000). International classification of diseases for oncology (3rd ed., pp. 98-100). Geneva: World Health Organization.

15. Brucker, P. S., Yost, K., Cashy, J., Webster, K., \& Cella, D. (2005). General population and cancer patient norms for the Functional assessment of cancer therapy-general (FACT-G). Evaluation \& the Health Professions, 28, 192-211.

16. Norman, G. R., Sloan, J. A., \& Wyrwich, K. W. (2003). Interpretation of changes in health-related quality of life: The remarkable universality of half a standard deviation. Medical Care, 41, 582-592.

17. Biggar, R. J., Chaturvedi, A. K., Goedert, J. J., \& Engels, E. A. (2007). HIV/AIDS Cancer Match Study (2007) AIDS-related cancer and severity of immunosuppression in persons with AIDS. Journal of the National Cancer Institute, 99, 962-972.

18. Holly, E. A., \& Lele, C. (1997). Non-Hodgkin's lymphoma in HIV-positive and HIV-negative homosexual men in the San Francisco Bay Area: Allergies, prior medication use, and sexual practices. Journal of Acquired Immune Deficiency Syndromes and Human Retrovirology, 15, 211-222.

19. Centers for Disease Control and Prevention (2005). Reported CD4+ T-lymphocyte results for adults and adolescents with HIV/ AIDS-33 states, 2005. HIV/AIDS Surveillance Supplemental Report, 11, 12. 